\title{
La Salud Bucal en la Percepción del Adolescente
}

\author{
Adolescents' perception of oral health
}

Cléa A. Saliba-Garbin, Artênio J. Isper-Garbin, Renato Moreira-Arcieri, Nemre A Saliba y Patricia E. Gonçalves

Departamento de Odontología Infantil y Social de la Facultad de Odontología de Araçatuba Universidad Estadual Paulista-UNESP, Brasil. cgarbin@ foa.unesp.br/pattysp03@yahoo.com.br

Recibido 17 Agosto 2008/Enviado para Modificación 2 Enero 2009/Aceptado 25 Febrero 2009

\section{RESUMEN}

Objetivo En la fase de la adolescencia, el individuo no siempre experimenta los mejores índices de salud y vitalidad. El objetivo de éste es evaluar la percepción del adolescente sobre las condiciones de su salud bucal, así como sobre los factores para su manutención y promoción.

Material y Método Fue aplicado un cuestionario semi estructurado a los adolescentes $(n=493)$ de la Fundación Mirim del Municipio de Araçatuba-SP, Brasil.

Resultados Entre los entrevistados, el 68,4 \% conceptuó salud bucal como higiene y el $16,2 \%$ como apariencia. Ya la percepción de los mismos cuanto a su Salud bucal fue un $53,6 \%$ buena y un $24 \%$ óptima. Con referencia a los medios para tener salud bucal, el 90,1\% relató la higiene bucal, pero apenas el 4,8\% de los adolescentes mencionó el cepillado de la lengua. La estética (62,9\%) fue uno de los principales motivos citados, cuanto a la importancia de los dientes. Fue observada una relación estadística significante entre la percepción de la Salud bucal regular y la visita al dentista hace menos de 6 meses $\left(X^{2}=30,75, p<0,05\right)$.

Conclusión Pese a que los adolescentes presentan una idea sobre Salud bucal, y sobre su manutención y promoción, estos temas deben ser más trabajados, principalmente por medio de Educación en Salud, para que esos adolescentes obtengan una buena Salud bucal y que ésta se mantenga por el resto de sus vidas. Además, ellos se vuelven multiplicadores de conocimientos para aquéllos que no tienen acceso a las mismas informaciones.

Palabras Clave: Odontología preventiva, salud bucal, educación en salud, adolescente (fuente: DeCS, BIREME).

\section{ABSTRACT}

Objective An individual does not always experience the best health and vitality indexes during her/his adolescence. This study evaluated young people's awareness of their oral health and the factors involved in dental maintenance and promotion

Material and Method Adolescents $(n=493)$ from the Mirim Foundation in the town of 
Araçatuba in the state of São Paulo, Brazil, were asked to fill in a semi-structured questionnaire.

Results $68.4 \%$ of the youngsters interviewed regarded oral health as referring to hygiene and $16.2 \%$ to physical appearance; $53.6 \%$ of the group perceived their oral health to be good and $24 \%$ optimum. $90.1 \%$ indicated oral hygiene when asked how oral health could be ensured; however, only $4.8 \%$ mentioned brushing their tongues. Physical appearance $(62.9 \%)$ was one of the main reasons stated as being important for teeth. A statistically significant relationship was observed between perception of regular dental and mouth health and having visited the dentist at least six months beforehand $\left(x^{2}=30.75 ; p<0.05\right)$.

Conclusion Even though the youngsters had a general idea regarding their oral health, its maintenance and promotion, better information should be made available regarding such topics and be approached through health education so that these adolescents can have good oral health for the rest of their lives, as well as becoming knowledge multiplying agents for those who do not have access to the same information.

Key Words: Preventative dentistry, oral health, health education, adolescent (source: MeSH, NLM).

$\mathrm{E}$ n el periodo de la adolescencia se produce la estructuración de la personalidad del individuo, a través de intensas transformaciones en los aspectos corporales, psicológicos y en sus relaciones. En ella el individuo experimenta los mejores índices de salud y vitalidad lo que le permitirá realizar sus tareas en la edad adulta (1). Pero es un período de riesgo para la Salud bucal. Sin embargo, se sabe que las enfermedades bucales prevalecientes pueden prevenirse con medidas de auto cuidado y de protección específica (2).

Por las razones anteriores es importante la realización de estudios con los adolescentes para que, a partir de sus vivencias, expectativas y visión del mundo, se pueda comprender mejor cómo se expresan y buscan ayuda, con el objetivo de auxiliar y orientar la creación de medidas más eficaces, y la planificación de acciones educativo-preventivas dirigidas a ese grupo $(1,3)$.

El objetivo de éste es evaluar la percepción del adolescente sobre las condiciones de su salud bucal, así como sobre los factores para su mantenimiento y promoción.

\section{MATERIAL Y MÉTODOS}

El proyecto fue aprobado por el Comité de Ética en Investigación de la Facultad de Odontología de Araçatuba-São Paulo, Brasil; bajo el n n 2006/01243, obedeciendo las normas éticas de la Resolución 196/96 y sus resoluciones 
complementares promulgadas por el Consejo Nacional de Salud del Ministerio de la Salud-Brasil, pues éste se desarrolló con la participación de seres humanos.

La población objeto de este estudio fue compuesta por $(\mathrm{n}=493)$ adolescentes, de los dos géneros, de 15 a 17 años de edad, matriculados en la Fundación Mirim de Araçatuba-SP, Brasil, institución que ofrece a los adolescentes cursos de capacitación para el mercado de trabajo. Ese número de individuos corresponde a la totalidad de alumnos de la institución en el año lectivo de 2006, dentro del perfil citado, y que estaban presentes en el aula en los días en que la investigación se llevó a cabo.

Antes que ingresaran los adolescentes a la institución, tras una explicación a todos los padres y/o responsables, se obtuvo el Término de Consentimiento Informado, en el que se informaba que el hijo iba a ser invitado a participar en una investigación sobre el tema de salud bucal. Esto se debió a que los adolescentes son considerados, ante la Resolución Brasileña no 196/96 sujetos vulnerables de investigación, pues presentan su autonomía reducida y, por lo que se requiere obtener el consentimiento. Además, en el cuestionario presentado a los adolescentes, se incluyó un breve encabezamiento, de acuerdo con las reglas relacionadas con la ética en investigación con seres humanos, aclarándoles sobre la participación voluntaria, el secreto de sus identidades (a pesar de no ser solicitada la identificación de los entrevistados), y la publicación de los datos obtenidos.

El instrumento de análisis utilizado fue un cuestionario semi estructurado, con seis preguntas subjetivas y cinco objetivas sobre Salud bucal, medidas de mantenimiento y su importancia. Se realizó una fase piloto para adecuación del instrumento.

Vale resaltar que no se realizó ninguna actividad educativa previa a la aplicación del cuestionario procurando obtener datos que demostrasen el real conocimiento sobre el tema evaluado de cada adolescente. La recolección de los datos fue realizada por un único examinador debidamente entrenado para la aplicación del instrumento. Los cuestionarios fueron respondidos individualmente por los adolescentes, en el aula, ante la presencia del investigador, para que éste pudiera dar algunas instrucciones necesarias para el diligenciamiento y aclarar eventuales dudas, tomando el cuidado de no influir en el contenido de las respuestas.

Después de la recolección de los datos, éstos fueron almacenados en un banco de datos del programa EPI INFO 3.2. Las respuestas a las preguntas abiertas fueron analizadas cualitativamente en cuanto a su contenido, a partir del 
Análisis de Contenido Temático (4). Éste define las categorías como rúbricas o clases, las cuales reúnen un grupo bajo un título general. Las respuestas fueron leídas para identificar los problemas apuntados y el conjunto de ellos formó una malla temática de categorías de análisis para la lectura transversal de todas las declaraciones, o sea, cada relato fue leído y clasificado según las categorías consideradas.

Las respuestas a las preguntas cerradas, fueron analizadas cuantitativamente y se realizó el test estadístico X-cuadrado, con significancia de $5 \%$. En todas las respuestas se examinó la distribución de frecuencias.

\section{RESULTADOS}

Entre los investigados, el $59 \%$ son del género femenino y el $41 \%$ del masculino. La edad de éstos varia entre 14 y 17 años, y la mayoría $(74,4 \%)$ presenta 15 años de edad.

En relación a los conceptos de Salud bucal, un 68,4 \% de los investigados la definieron como higiene, un 16,2\% como apariencia y un $15,5 \%$ como ausencia de dolencias bucales (Tabla 1).

Tabla 1. Distribución de la frecuencia absoluta y relativa de las categorías de Salud bucal según los adolescentes ${ }^{a}$. Araçatuba-SP, 2007

\begin{tabular}{lcc}
\hline $\begin{array}{l}\text { Higiene-"Salud bucal seria pasarse el hilo dental, cepillarse, } \\
\text { etc..." }\end{array}$ & 292 & 68,4 \\
$\begin{array}{l}\text { Apariencia-"Cuando sus dientes están buenos y bonitos" } \\
\begin{array}{l}\text { Ausencia de dolencias bucales-"Salud bucal es estar sin caries } \\
\text { ni otras enfermedades" }\end{array}\end{array}$ & 69 & 16,2 \\
$\begin{array}{l}\text { Cuidado profesional-"Es estar yendo al dentista regularmente } \\
\text { previniendo asi las enfermedades" }\end{array}$ & 54 & 12,6 \\
$\begin{array}{l}\text { Dientes, encías saludables-"Salud bucal es tener los dientes y } \\
\text { la encia saludable, sin problemas" }\end{array}$ & 36 & 8,4 \\
$\begin{array}{l}\text { Salud de la boca-"Es la salud de nuestra boca, nuestros } \\
\text { dientes y todos los demás componentes de la boca" }\end{array}$ & 25 & 5,9 \\
$\begin{array}{l}\text { Alimentación saludable-"Debemos tomar comidas } \\
\text { saludables para no dañarnos los dientes" }\end{array}$ & 4 & 0,9 \\
\hline
\end{tabular}

a 66 adolescentes investigados no respondieron la pregunta; b. es posible que la misma respuesta tuviera más de una categoría

La percepción de los adolescentes sobre su salud bucal fue buena en un $53,6 \%$, un $24 \%$ óptima, un 20,6\% regular, un 1,6\% mala y un 0,2 \% pésima. 
Referente a los medios para lograr una salud bucal, las principales respuestas consistieron en higiene bucal $(90,1 \%)$ y cuidado profesional $(42,7 \%)$ (Tabla 2$)$.

Tabla 2. Distribución de la frecuencia absoluta y relativa de las categorías de medios para tener Salud bucal según los adolescentesa ${ }^{a}$.

\begin{tabular}{lcc}
\multicolumn{1}{c}{ Araçatuba-SP, 2007 } & \\
\hline \multicolumn{1}{c}{ Categorias } & $\mathrm{N}^{\mathrm{b}}$ & $\%$ \\
\hline Higiene bucal-"Cepillar los dientes, pasar hilo dental, flúor, etc. " & 437 & 90,1 \\
Cuidado profesional-"Ir a un dentista siempre, para ver si hay & 207 & 42,7 \\
algo malo en la boca o alguna cosa que pueda afectar la salud" & 27 & 5,6 \\
Cuidado con la alimentación-"No comer golosinas" & 5 & 1 \\
Evitar hábitos malos-"No fumar" & 1 & 0,2 \\
$\begin{array}{l}\text { Otros-"Mantenernos siempre informados sobre nuestra salud } \\
\text { bucal" }\end{array}$ & & \\
\hline $\begin{array}{l}\text { a. } 8 \text { adolescentes investigados no respondieron la pregunta; b. Es posible que la misma respuesta } \\
\text { tuviera más de una categoría }\end{array}$ &
\end{tabular}

$\mathrm{Al}$ describir sobre la categoría «higiene bucal» se observó que el 79,1\% de los entrevistados relataron el cepillado de los dientes, el 42,2 \% el uso del hilo dental. Se verificó que apenas el 4,8 \% de los adolescentes citaron el cepillado de la lengua como higiene.

Todos los que respondieron afirmaron que los dientes son importantes para los adolescentes. Y sobre el principal motivo por el cual aquellos eran importantes, fue citada la estética $(62,9 \%)$ (Tabla 3$)$.

Tabla 3. Distribución de la frecuencia absoluta y relativa de las categorías de la importancia de los dientes según los adolescentes ${ }^{a}$.

Araçatuba-SP, 2007

\begin{tabular}{lcc}
\hline \multicolumn{1}{c}{ Categorías } & $\mathrm{N}^{b}$ & $\%$ \\
\hline $\begin{array}{l}\text { Estética-"Porque si usted no tiene una buena sonrisa, y los } \\
\text { dientes blancos, usted se vuelve una persona fea" }\end{array}$ & 288 & 62,9 \\
$\begin{array}{l}\text { Masticación-"Porque ellos tienen la función de triturar los } \\
\text { alimentos para ayudar en la digestión" }\end{array}$ & 260 & 56,8 \\
$\begin{array}{l}\text { Convivencia social-"Porque si no cuidamos nuestros dientes, } \\
\text { ellos se van dañando y acabando, entonces no logramos } \\
\text { conseguir un novio ni amigos" }\end{array}$ & 49 & 10,7 \\
$\begin{array}{l}\text { Fonación-"Sin ellos no logramos hablar bien" } \\
\text { Salud General-"Porque hacen parte de nuestro cuerpo" }\end{array}$ & 30 & 6,6 \\
$\begin{array}{l}\text { Mercado de trabajo-"Porque es una tarjeta postal para un } \\
\text { óptimo empleo" }\end{array}$ & 18 & 3,9 \\
$\begin{array}{l}\text { Evitar mal hálito-"Dientes bonitos le dan un hálito bueno a la } \\
\text { persona" }\end{array}$ & 15 & 3,3 \\
$\begin{array}{l}\text { Aspectos afectivos-"Porque son bienes preciosos que } \\
\text { tenemos" }\end{array}$ & 2 & 0,4 \\
\hline \begin{tabular}{l} 
a. 35 adolescentes investigados no respondieron la pregunta; b. Es posible que la misma respuesta \\
\hline
\end{tabular}
\end{tabular}

tuviera más de una categoría 
Sobre cuáles dientes eran más importantes para los adolescentes, más de la mitad de la muestra $(59,9 \%$ ) apuntó todos los dientes, aunque el 21,8 \% mencionó los dientes de adelante.

En lo que se refiere a la percepción de los adolescentes en cuanto a sus dientes se observó que más de la mitad los consideró como buenos (52\%), óptimos $(23,1 \%)$, regulares $(22,5 \%)$. Poquísimos los caracterizaron como malos $(1,8 \%)$ y pésimos $(0,6 \%)$.

En relación con la última visita de los adolescentes al dentista, el 29,8 \% habían estado hacía 1 mes, el 22,3\% hacía entre 1 a 6 meses, el 23,9 \% de 6 meses a 1 año y el 22,4 \% habían estado hacía más de un año. De los entrevistados apenas 8 adolescentes $(1,6 \%)$ nunca habían recibido cualquier tipo de atención o tratamiento odontológico.

$\mathrm{Al}$ relacionar la percepción del adolescente sobre su salud bucal y la visita al cirujano-dentista, se observó una relación estadística significativa entre la percepción de la salud bucal regular y la visita al dentista hace menos de 6 meses $\left(X^{2}=30,75, p=0,05\right)$.

De los locales que los adolescentes obtuvieron tratamiento odontológico, un 48,9\% fue en la Unidad Básica de Salud-UBS, un 36,5\% en el Consultorio Particular, un 12,8 \% en la Facultad de Odontología de Araçatuba-UNESP, un $22,3 \%$ en la Escuela Pública, un 6,7 \% en el Convenio (seguro privado) y un 3,7 $\%$ en otros locales.

Sobre si los entrevistados habían recibido alguna información sobre Salud bucal, apenas el $8 \%$ no habían recibido. Entre los que habían recibido, el principal lugar fue la escuela $(79,1 \%)$, después, con menor porcentaje, el consultorio odontológico (7,8 \%), la Unidad Básica de Salud-UBS (5,6 \%), la Facultad de Odontología de Araçatuba-UNESP (2,5\%), la familia, la Tele, los folletos (1 \% cada uno), y otros lugares $(2,0 \%)$.

\section{DISCUSIÓN}

Las acciones relacionadas con Salud bucal en la adolescencia deben desarrollarse para proporcionar al adolescente una mejor calidad de vida, no sólo en esa etapa, sino para el resto de su vida. 
Referente a los conceptos de Salud bucal, el 12,6 \% de los adolescentes mencionó el cuidado profesional como tal. Sin embargo, los autores relatan que el impulso de buscar atención odontológica por los adolescentes está directamente relacionado con la participación e interés de los responsables en la manutención de la Salud bucal de ellos, y también con la propia vanidad de los mismos (5).

En el estudio con adolescentes chilenos de edad entre 12 a 20 años, percibieron que la estética es la principal razón para el cuidado bucal, concordando con $(16,2 \%)$ de los investigados, que conceptuaron la salud bucal como la apariencia (6). En el nivel psicosocial, una boca saludable garantiza la manutención de la buena apariencia, de la expresión y de la comunicación interpersonal, siendo así, un factor de importancia mayor en la preservación de la auto-estima (7).

Sobre la percepción de la Salud bucal, en el Proyecto Salud bucal Brasil 2003(8), se observó que cerca del 44,6 \% de los adolescentes clasificaron su Salud bucal como buena, el $34 \%$ como regular y el 5,8 \% como óptima. En el presente trabajo se obtuvo un mayor porcentaje cuanto a la clasificación buena $(53,3 \%)$ y óptima $(24 \%)$.

En cuanto a la visita al cirujano-dentista se observó que la mayoría $(52,1 \%)$ había estado hacía menos de seis meses. Sin embargo, fue estadísticamente comprobado que aquellos adolescentes que habían estado hacía menos de seis meses en el dentista caracterizaron su salud bucal como regular. Eso puede ser justificado, conforme lo observado en el estudio (9), que los factores asociados a la mejor auto-evaluación de la Salud bucal estarían directamente relacionados al mayor número de visitas al dentista, necesidad auto-referida de tratamiento odontológico, presencia de mayor número de dientes permanentes, menor número de dientes cariados o restaurados, mejor condición periodontal (10-12).

Autores (13) relatan que la salud bucal se determina por la dieta, higiene, hábito de fumar, uso de alcohol, tensión y trauma, y enfatizan la necesidad de difundir una política de comidas saludables en escuelas, como medio efectivo de promover la Salud bucal, pues se sabe que la alimentación de los adolescentes en la escuela es cariogénica, debido a la gran oferta de bocadillos y gaseosas.

En cuanto a los adolescentes que describieron la higiene bucal como cepillado de los dientes (90,1\%), el estudio (14) con estudiantes observó que ninguno relacionó como factor etiológico de la carie dental la asociación entre la dieta y la higiene. Posiblemente, eso pueda ser explicado por la idea de los alumnos de que el cepillado y la higiene bucal son la misma cosa. 
En cuanto a los medios de higiene, un $84,4 \%$ de los investigados mencionó el cepillado de los dientes y apenas el $45 \%$ el uso del hilo dental. La motivación que lleva a los adolescentes a realizar sus hábitos de higiene, es la cuestión del mal hálito, o la verguienza, factores que están asociados al contacto con los otros colegas, amigos (as) o novio (a) (15).

La resistencia al uso del hilo dental se observa entre los jóvenes, pues apuntan la dificultad de su uso por la habilidad exigida, y la necesidad de entrenamiento, además de la pereza en adoptarlo como recurso de rutina. Así, el uso del hilo ocurre en situaciones especiales, como fiestas de fin de semana (16).

Se puede deducir que mientras el hábito del cepillado es usual en las familias de los adolescentes, el uso del hilo dental es menos frecuente (17). Eso también puede ser observado en este estudio cuanto al cepillado de la lengua que fue mencionado por apenas el 4,5\% de los entrevistados como medio de higiene bucal, pues se sabe que la lengua, cuando no cepillada, se vuelve un local de procreación y diseminación de microorganismos que fatalmente estarán en contacto con todo el medio bucal.

Sobre la percepción de los dientes, las relaciones entre la estética y la salud bucal, particularmente la Salud dental, son complejas y abarcan dimensiones sociales, culturales y psicológicas (17), con eso se entiende por qué la mayoría de los adolescentes citó como importancia de los dientes, la estética (62,9 \%). Eso fue observado en otros estudios $(7,18)$, pues la estética fue la principal razón por la que el adolescente buscaba la atención odontológica.

Se sabe que el joven está dirigido para los aspectos estéticos de su cultura como forma de comparar su cuerpo con un modelo. Teniendo su imagen perjudicada, el adolescente puede perder su auto-estima haciendo con que esto interfiera en sus relaciones personales, además de eventualmente producir sentimientos de inferioridad, concordando con el 10,7 \% de los adolescentes entrevistados en el presente trabajo, en el que relataron que los dientes son importantes para la convivencia social, y para las entrevistas de empleo $(3,3 \%)$.

Según el Proyecto Salud bucal Brasil 2003 (8), la percepción de los adolescentes cuanto a la apariencia de sus dientes y encías fue un $49 \%$, un $28,7 \%$, un $7,9 \%$, un $6,7 \%$ y un $3 \%$ como buena, regular, mala, óptima y pésima respectivamente. En este estudio las categorías regular $(22,5 \%)$ y buena (52\%) presentaron un porcentaje semejante al del proyecto. Aunque los resultados malos y pésimos tuvieron valores bien inferiores a los encontrados por el proyecto, el 
resultado óptimo $(23,1 \%)$ ocurrió cuatro veces mayor al encontrado por el proyecto brasileño.

Se constató que cerca del $14 \%$ de los adolescentes brasileños nunca fue al dentista, el 48,6 \% habían ido hacía menos de un año y el $38 \%$ hacía más de un año(8). Se observó un valor inferior en cuanto a los adolescentes que nunca fueron al dentista $(1,6 \%)$. Pero, el $76 \%$ había ido hacía menos de un año y el $22,4 \%$ hacía más de un año. Estos últimos porcentajes resultan de la presencia de la Facultad de Odontología en el municipio de Araçatuba- UNESP, que favorece el acceso de ellos al tratamiento odontológico, como también de la gran oferta por el sistema público y particular del municipio.

La escuela fue citada por un 79,1\% de los alumnos como local donde obtuvieron informaciones sobre Salud bucal, lo que reafirma el propósito del Ministerio de la Salud Brasileño (19) que considera la escuela un ambiente educacional y social propicio para trabajar conocimientos y mudanzas de comportamiento. Además, los programas educativos promovidos en escuelas no perjudican a los niños cuanto a su aprendizaje, y los animan a tener Salud bucal por mucho tiempo, siendo un refuerzo para la manutención de comportamientos de Salud a fin de promover la Salud individual de grupos o de la comunidad (20-22).

El cirujano-dentista debe ser consciente de la responsabilidad de educar a sus pacientes para la Salud bucal, principalmente a los jóvenes, pues en un estudio (16) observaron que apenas el 4,9 \% de los adolescentes analizados relató a los familiares como principal fuente de informaciones sobre dolencias bucales, así como fue encontrado en este estudio (1\%). Ello enfatiza la necesidad de ese adolescente de hacerse un agente multiplicador sobre salud bucal en el entorno en que vive, más allá de la escuela, llevando a las otras personas, que no tuvieron el mismo acceso que él a la información correcta y adecuada

\section{REFERENCIAS}

1. Palazzo LS, Bérla JU, Tomasi E. Adolescentes que utilizan servicios de atención primaria: ¿Cómo viven? ¿Por qué buscan ayuda y cómo se expresan? Cad Saúde Publica 2003; 19: $1655-1665$.

2. Valente MSG. Adolescência y salud bucal. Adolesc Latinoam 2004; 98: 170-174.

3. Petry PC, Pretto SM. Educação e Motivação em Saúde Bucal. En: Kriger, L. ABOPREV: Promoção de Saúde Bucal. 3. ed. São Paulo: Artes Médicas; 2003 p. 372-385.

4. Bardin L. Análise de Conteúdo. Lisboa: Editora Persona; 1994.

5. Colares V, Farias JN, Bezerra LC, Oliveira MM. Avaliação da Saúde Bucal de adolescentes de Recife-Pernambuco- Brasil. Rev Bras Ciênc Saúde 2002; 6: 217-224. 
6. Misrachi LC, Arellano OM. Conductas y factores determinantes en salud oral de los adolescentes. Rev Chil Pediatr 1995; 66: 317-322.

7. Weyne SC. A Construção do paradigma de promoção de Saúde: um desafio para as novas gerações. Em: Kriger, L. ABOPREV: Promoção de Saúde Bucal. 3. ed. São Paulo: Artes Médicas; 2003. p.1-26.

8. Brasil. Ministério da Saúde. Projeto SB Brasil 2003. Condições de saúde bucal da população brasileira 2002-2003. Resultados principais. Brasília: Coordenação Nacional de Saúde Bucal; 2004.

9. Matos DL, Lima-Costa MF. Auto-avaliação da saúde bucal entre adultos e idosos residentes na região Sudeste: resultados do projeto SB- Brasil, 2003. Cad Saúde Pública 2006; 22:1699-1707.

10. Atchison KA, Gift HC. Perceived oral health in a diverse sample. Adv Dent Res. 1997; 11:272280.

11. Gift HC, Atchison KA, Drury TF. Perceptions of the natural dentition in the context of multiple variables. J Dent Res 1998; 77: 1529-1538.

12. Matthias RE, Atchison KA, Lubben JE, De Jong F, Schweitzer SO. Factors affeting self-ratings of oral health. J Public Health Dent 1995; 55: 197-204.

13. Sheimam A, Watt RG. The commom risck factor approach: a racional basis for promoting oral health. Community Dent Oral Epidemiol 2000; 399-406.

14. Pinto LR, Bonan RF, Garcia PPNS. Conhecimento sobre Cárie e Doença Periodontal: Avaliação de Adolescentes Pertencentes à Rede Privada de Ensino. Rev Odontol da UNESP 2004; 33:137-142.

15. Flores EMTL, Drehmer TM. Conhecimentos, percepções, comportamentos e representações de Saúde e doença Bucal dos adolescentes de escolas públicas de dois bairros de Porto Alegre. Ciênc Saúde Coletiva 2003; 8: 743-752.

16. Macgregor IDM, Balding JW, Regis D. Flossing behavior in English adolescents. J Clinical Periodontol 1998; 25: 291-296.

17. Baldwin DC. Appearance and aesthetics in oral health. Community Dent Oral Epidemiol 1980; 8: 244-256.

18. Misrachi LC, Arellano OM. Adolescentes: factores predisponentes, facilitadores y reforzadores de conductas en salud oral. Odontol chil 1996; 44: 23-29.

19. Brasil. Ministério da Saúde. Criança, adolescente e adulto jovem. Brasília. Documento de referência para o trabalho de prevenção das DST, Aids e drogas. Coordenação Nacional de DST e Aids; 1997.

20. Teng O, Narksawat K, Podang J, Pacheun O. Oral health status among 12-year-old children in primary schools participating in an oral preventive school program in Phnom Penh City, Cambodia, 2002. Southeast Asian J Trop Med PublicHealth 2004; 35:458-462.

21. Vignavajah $\mathrm{S}$. Oral health knowledge and behaviours and barriers to dental attendance of school children and adolescents in the Caribbean island of Antigua. Int Dent J 1997; 47: 167-172.

22. Oliveira ER, Narendran S, Williamson, D. Oral health knowledge, attitudes and preventive practices of third grade school children. Pediatr Dent 2000; 22: 395-400. 